\title{
Increased prevalence of large genomic imbalances among children with chronic kidney disease
}

$\mathrm{C}$

hildren with chronic kidney disease (CKD) have an excess burden of large genomic imbalances that are not suspected based on standard clinical examinations, according to a new study. Researchers Ali Gharavi, Miguel Verbitsky and colleagues suggest that these findings have important implications for the diagnosis and personalized management of paediatric kidney disease.

"CKD in children is associated with significant morbidity and mortality, but we often cannot make a precise diagnosis of the underlying disease because of late referral or overlap in the clinical presentation of different disorders," says Gharavi. "We previously found that rare genomic structural variants (mutations involving deletions or duplications of DNA) are very common in children with CKD owing to congenital kidney malformations, and can provide a precise diagnosis of kidney disease. In our new study we investigated whether similar genomic lesions are present in patients with many different forms of CKD."

In collaboration with the $\mathrm{CKiD}$ investigators, Gharavi, Verbitsky and colleagues used high-density chromosome microarrays to analyse the genomes of 419 unrelated North American children with mild to moderate CKD enrolled in the CKiD prospective cohort study. This technique enables detection of submicroscopic chromosomal abnormalities that would not be identified using traditional karyotyping. "As many DNA deletions or duplications can be benign, we compared the CKiD samples to chromosomal microarray data from a large multiethnic cohort of 21,575 adults and children without kidney disease, and searched for DNA abnormalities that were either diagnostic for known genetic disorders or were very rare and potentially a cause of disease," explains Verbitsky.

The researchers found pathogenic and likely pathogenic DNA imbalances in $4.5 \%$ and $2.9 \%$ of children in the CKiD cohort, respectively. By contrast, only $0.5 \%$ of individuals in the control group had a pathogenic DNA imbalance (OR 10.8, $\left.P=6.1 \times 10^{-20}\right)$. This difference in prevalence remained statistically significant after adjustment for sex and ethnicity and when adult controls were excluded from the analysis. In some patients the genomic findings confirmed the clinical diagnosis of kidney disease (for example, deletion of the cystinosin gene in those with cystinosis), but in the majority of cases the genomic lesion was not suspected based on the clinical evaluation and its detection reclassified or refined the previous diagnosis. In the clinical setting these findings would have informed decisions for genetic counselling, additional testing, treatment and follow-up.

"Many of the disorders we identified in children with CKD potentially affect metabolic parameters, neurocognitive and/or behavioural function and growth, so warrant additional work-up and/ or surveillance," comments Gharavi. "These data suggest that in some patients, comorbidities might not be secondary to impaired kidney function, but could stem from a common genetic aetiology." Moreover, the researchers identified genomic disorders across the whole spectrum of paediatric CKD. "This finding suggests that in many cases we cannot identify and distinguish genetic kidney disorders by clinical evaluation. It might also indicate that the presence of rare pathogenic genomic variants could be a common denominator conferring genetic risk for CKD."

Gharavi, Verbitsky and colleagues conclude that a subset of patients with CKD have undiagnosed genetic disorders, and that implementation of genetic testing will enable precise diagnosis of kidney disease and the development of personalized work-up and therapy. "We need to validate our findings in an independent CKD population before we can recommend routine genetic testing in all children with CKD. However, based on the findings of this study and our previous data, it is clear that children with CKD due to kidney malformations (renal aplasia, hypoplasia or dysplasia) have the highest risk of having a genomic imbalance. These patients should be considered for DNA microarray analysis now, and for genome sequencing in the near future."

Finally, the researchers highlight the potential wider implications of their findings for the use of genomic diagnostics to inform patient care. "Our analysis based on data obtained by the CKiD study serves as proof of principle that nephrology may be ideally positioned to spearhead the adoption of genomic diagnostics in clinical practice. Perinatal imaging and laboratory tests are routinely performed to assess kidney development and function, and detection of CKD could become an indication to search for genomic imbalances to achieve personalized medical interventions at an early stage."

Ellen F. Carney

Original article Verbitsky, M. et al. Genomic imbalances in pediatric patients with chronic kidney disease. J. Clin. Invest. doi:10.1172/JCl80877 